\title{
LiposomalAmphotericin-BinSalineShowsPromisingEfficacy against Candida auris compared to Azoles, Echinocandins and Other Amphotericin-B Formulations in Dextrose and Deoxycholate Suspension
}

\author{
Vijay Pancholi* \\ Department of Pathology, The Ohio State University College of Medicine, Columbus, USA
}

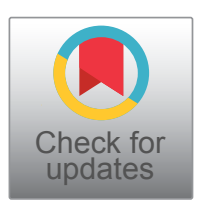

*Corresponding author: Vijay Pancholi, PhD, Department of Pathology, The Ohio State University College of Medicine,

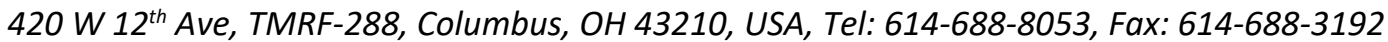

\begin{abstract}
Fungal infection caused by Candida auris is now a global crisis. However, its multidrug-resistant nature and onerous identification have left limited therapeutic options resulting in 30-60\% mortality. Amphotericin-B (AmB), a broad-spectrum and a potent fungicidal agent with a rare resistance pattern, is considered a gold-standard antifungal drug. Yet, its antifungal potential is not fully recognized under the pretext of its potential nephrotoxicity. Liposomal AmB formulations are safer than AmB-Deoxycholate. The Liposomal AmB in Saline (Fungisome) has no nephrotoxicity. In this study, seven antifungals, including three Echinocandins (Caspofungin, Micafungin, Anidulafungin), one azole (Posaconazole), and three AmB formulations (AmBDeoxycholate, and Liposomal AmBisome ${ }^{\circledR}$ and Liposomal FUNGISOME $^{\circledR}$ in saline) were examined for their efficacy against ten MDR $C$. auris and one C. glabrata strains employing broth dilution antifungal susceptibility tests (BDAFST) to assess their potential therapeutic applicability. $C$. auris and C. glabrata strains showed variable susceptibility patterns to Echinocandins and Posaconazole. Compared to these antifungal agents, all Candida strains were susceptible to FUNGISOME ${ }^{\circledR}$ and showed 8-32-fold lower MIC than AmBisome ${ }^{\circledR}$ and AmB-Deoxycholate, which were well within the susceptible breakpoint-range. Given the known toxicity of different antifungal agents and their cost-effectiveness, these findings underscore the importance of incorporating liposomal AmB formulations in the conventional BD-AFST to reflect correct AmB-resistance patterns. These changes will allow making an educated decision in recommending a therapeutically promising antifungal agent to treat $C$. auris and other fungal infections during clinical practices in hospital setups.
\end{abstract}

\section{Introduction}

Fungal infections in general and Candida spp infections, in particular, are a major cause of nosocomial invasive disease and a severe concern to human health globally [1-3]. These infections are typically associated with predisposing immune-deficient status, prolonged hospitalization, broad-spectrum antibiotics, and prophylactic use of antifungal agents such as fluconazole $[4,5]$. Candidemia is the most common form of invasive candidiasis. However, Candida's invasiveness does not necessarily represent its detection in the heart, kidney, and other internal organs because of its absence in the blood $[6,7]$. Hence, CDC estimates the actual burden of invasive candidiasis might be twice as high as the estimate for candidemia [8].

Although C. albicans is the principal agent of nosocomial infection, non-albicans Candida $s p$ infections have been associated with higher mortality and drug resistance in the last decades $[4,6,7]$. Of these non-albicans species, $C$. auris is a rapidly growing global public health challenge. It is the most serious, emerging, and a multidrug-resistant fungal pathogen that is rapidly spreading worldwide and causing mortality in 30-60\% of the infected patients [9-17]. The earliest Candida isolate, identified in 2011 as C. auris, was taken from a bloodstream sample of a patient from Korea in 1996 [18]. C. auris, as such, was first reported in 2009 from ear

Citation: Pancholi V (2021) Liposomal Amphotericin-B in Saline Shows Promising Efficacy against Candida auris compared to Azoles, Echinocandins and Other Amphotericin-B Formulations in Dextrose and Deoxycholate Suspension. Int Arch Med Microbiol 4:014. doi.org/10.23937/2643-4008/1710014 Accepted: October 23, 2021: Published: October 25, 2021

Copyright: (C) 2021 Pancholi V. This is an open-access article distributed under the terms of the Creative Commons Attribution License, which permits unrestricted use, distribution, and reproduction in any medium, provided the original author and source are credited. 
cultures (and hence "auris") of one patient in Japan [19] and 15 patients from University Hospitals South Korea [20]. In a decade since then, the infection has spread to all six continents $[10,14-17,21,22]$. Epidemiological data have shown that India and Pakistan have the highest cases of $C$. auris infections, primarily reported in overcrowded and tertiary care trauma hospitals $[10,17,23]$. Phylogenetic analysis of the globally isolated C. auris and related fungal strains has revealed that the climate change/global warming-related swamps, the widespread use of azoles, and the birds' migrationmediated transmission may have contributed to the simultaneous emergence of $C$. auris in three different continents $[17,24]$.

High mortality caused by invasive and systemic mycosis in general, the emergence of multidrugresistant invasive $C$. auris infections, and limitations of early detection and correct identification of $C$. auris together have left the health system with limited therapeutic options. Hence, the $C$. auris infection is the most challenging unmet medical need $[11,16,21,25,26]$. This situation is precipitously felt in the ongoing Covid-19 infection pandemic $[27,28]$. Typical $C$. auris isolates display reduced susceptibility to azoles, polyenes, echinocandins, and fluconazole [3,11,14,16,29-32]. Several studies have also shown that $C$. auris isolates also display increased resistance/reduced susceptibility patterns to the second generation and improved azoles antifungals, such as voriconazole, posaconazole, itraconazole, and isavuconazole $[30,31,33]$. Some of the biggest challenges in antifungal/anti- $C$. auris infections therapy regimens are i) a limited antifungal spectrum, ii) inadequate potency, iii) significant toxicity, and iv) the rapid onset of preexisting antifungal drug resistance even before it is marketed $[3,30,34]$. In many instances, the use of these antifungal agents results in poor treatment outcomes in the absence of apparent alternatives $[12,35]$. Amphotericin- $B$, a polyene macrolide antibiotic, was discovered in the 1950s with its potent broadspectrum antifungal [36] and antileishmanial activities [37]. Because of the absence of its genetically confirmed resistance in 60 years of its clinical use, Amphotericin-B has been the mainstay for treating the highly fatal group of fungal infections [36].

Amphotericin-B, despite having the merit of fungicidal potency [36], poses dose-limiting nephrotoxicity leading to renal failure [38-40]. Two distinctly different Amphotericin-B liposomal formulations have been developed to overcome its inherent nephrotoxicity. These liposomal formulations are ideal antifungal agents for empirical, prophylactic, and case-targeted systemic and invasive mycosis [41-43]. Evolving Amphotericin-B parenteral formulations from a deoxycholate suspension to lipid complex [44] and then to liposomal preparations have led to incremental lowering of dose-limiting toxicity and consequent improved clinical outcomes [41,45-47]. However, the therapeutic usage of liposomal formulations of Amphotericin-B in C. auris has not been, hitherto, examined. The liposomal Amphotericin-B preparations, proven for higher antifungal efficacy [48], are typically not included for antifungal susceptibility testing for Candida spp [49-53], including C. auris $[26,32,54,55]$. As such, significant controversy and difficulty in judging the breakpoint of Amphotericin-B for Candida spp exist, and specific breakpoints have not been proposed due to interlaboratory variations [49-53]. The applicability of Amphotericin-B therapy, in general, whether it would pose dose-dependent nephrotoxicity, is judged based on the antifungal susceptibility pattern of Candida spp to Amphotericin-B-deoxycholate [56]. Although the most accurate way to determine antifungal agents, including Amphotericin-B susceptibility, is the CLSI recommended broth dilution method [57], routine laboratories heavily rely on additional methods, such as E-test and Vitek-2 systems, to report identification and/or AFST for yeast. A recent report on comparative findings on AFST of 102 C. auris strains against Amphotericin-B using the CLSI-Broth microdilution, Vitek-2, and E-test methods showed inconsistent MIC results [26]. In this study, Vitek-2 and E-test revealed high MICs and low MICs, respectively, for the same $C$. auris strains. Thus, in general, $C$. auris antifungal susceptibility patterns are interpreted using breakpoints established for other Candida species [17]. The cutoff points for susceptibility for C. auris against Amphotericin-B, is carried out using Amphotericin-B deoxycholate suspension [32]. Based on such inconsistent results, MDR $C$. auris infections are presumed to be recalcitrant to Amphotericin-B, including the improved yet untested liposomal Amphotericin-B in the AFST. This practice is followed even though the resistance to Amphotericin-B in Candida spp is rare $[36,58]$ and the mechanism of this resistance is presently not fully understood for $C$. auris [59]. To that end, another study has, however, shown that the CLSI-based MIC of a novel liposomal Amphotericin-B in Saline (FUNGISOME ${ }^{\circ}$ ) is $2-16$ folds lower than Amphotericin-B deoxycholate for several species of yeast and molds, which did not include $C$. auris [60]. These studies underscore the fact that the AFST for Amphotericin-B against yeast and molds in general and $C$. auris, in particular, should be revisited.

Presently, there is no information on whether $C$. auris strains generally show differential susceptibility patterns to these improved preparations of liposomal Amphotericin-B compared to variant forms of available Amphotericin- $B$ formulations and other antifungal agents. This existing knowledge gap prompted us to investigate and compare the susceptibility patterns of 10 C. auris and one C. glabrata against seven antifungal drugs, including three each of different Amphotericin-B formulations (Amphotericin-Bdeoxycholate, Liposomal Amphotericin-B: AmBisome ${ }^{\circ}$, and Liposomal Amphotericin-B in Saline: FUNGISOME ${ }^{\circ}$ ), 
and echinocandins (Caspofungin, Micafungin, and Anidulafungin), and one azole, Posaconazole. The present study showed that the liposomal preparation of Amphotericin-B in Saline, FUNGISOME ${ }^{\circledR}$, displayed uniform higher efficacy against all MDR $C$. auris strains.

\section{Materials and Methods}

\section{Isolates and growth conditions}

A total of 10 multidrug-resistant $C$. auris strains CDC-0381-0390), and one C. glabrata (CDC-0317) were obtained from the antibiotic-resistant strain collection of CDC. All strains were grown and maintained on Sabouraud's dextrose agar or broth.

\section{Antifungal agents}

Three formulations of Amphotericin-B, Micafungin, Caspofungin, Anidulafungin, Posaconazole were obtained as indicated. Amfocare ${ }^{\mathrm{TM}}$ (Colloidal dispersion of Amphotericin-B in deoxycholate, Bangalore Pharmaceutical), Liposomal AmBisome (Mylan), Liposomal in Saline (FUNGISOME ${ }^{\bullet}$, Lifecare Innovations), Caspofungin (Casfung ${ }^{\mathrm{TM}}$, Glenmark), Micafungin (Micona $^{\mathrm{TM}}$, Glenmark), Anidulafungin (Andulfa ${ }^{\mathrm{TM}}$, Gufic /Intas), and Posaconazole (Posatral ${ }^{\mathrm{TM}}$, Mylan). All antifungal agents other than those obtained from Sigma were of pharmaceutical grades and were reconstituted in saline, PBS, or distilled water per the respective manufacturers' instructions. FUNGISOME ${ }^{\circledR}$ preparation was sonicated for $45 \mathrm{~min}$ using a temperature-controlled water sonicator bath per the manufacturer instructions before use.

\section{Antifungal susceptibility test (AFST)}

AFST was carried out using the Clinical and Laboratory Standard Institute broth microdilution method (CLSIBMD)-M270 A3-guidelines [57]. Briefly, RPMI-1640 medium with glutamine without bicarbonate containing 0.165M MOPS pH 7.0 (Sigma) was used for 2-fold serial dilution $(32 \mu \mathrm{g} / \mathrm{ml}$-to $0.0156 \mu \mathrm{g} / \mathrm{ml})$ and also to dilute the stock solutions of antifungal agents in microtitre plates. Overnight cultures of Candida species grown on Sabouraud's dextrose agar were suspended in RPMI broth and adjusted to O.D. ${ }_{620 \mathrm{~nm}}$ of 0.1 (equivalent to McFarland Standard 0.5). $100 \mu$ l of each culture was added to the individual wells containing $100 \mu$ l of serially diluted antifungal agents. Antifungal agent- and Candida species-free controls were included as blank background readings. Similarly, wells containing Candida species without any antifungal agents were treated as no (0\%) inhibition. Microtiter plates with tests and controls samples were incubated at $35^{\circ} \mathrm{C}$ for $24 \mathrm{~h}$. All experiments were carried out in three individual biological replicates. Growth inhibition of individual Candida species against different antifungal agents was observed visually as well as spectrophotometrically (PolarStar Galaxy, BMG, O.D. $\left.{ }_{620 \mathrm{~nm}}\right)$. Average background readings obtained from 6-12 wells/plate were subtracted from the test results for further calculations. Minimum inhibition concentration (MIC) for all Amphotericin-B variants was defined as the lowest concentration at which $100 \%$ inhibition was achieved (both visually and by culture confirmation). The MIC endpoints were defined as the lowest concentration that caused $50 \%$ growth inhibition relative to growth control (No inhibition) for the rest of the antifungal agents. Percent Inhibition of Candida species by individual antifungal drugs at different concentrations was calculated using formula $=100 \mathrm{X}$ (1-O.D. of test sample/average O.D. of control wells receiving respective Candida $\mathrm{sp}$.). Inhibition curves for each strain for all antifungals were plotted using GraphPad prism 6, and $50 \%$ and $90 \%$ inhibition values were determined. Fungicidal activity of echinocandins (Micafungin and Anidulafungin) was determined as described above for Amphotericin-B based on optical density as well as culture negativity.

\section{Results}

In the present study, seven different antifungal agents against 11 different Candida species, 10 of which belonged to multidrug-resistant $C$. auris, and one $C$. glabrata were subjected to AFST analysis. $\mathrm{MIC}_{50}$ and $\mathrm{MIC}_{90}$ of all antifungals except Amphotericin-B formulations are shown in Table 1 and three Amphotericin-B formulations in Table 2 based on inhibitory concentration $50\left(\mathrm{IC}_{50}\right)$ and $\mathrm{IC}_{90}$ of individual strain. As shown in Table 1 , all $C$. auris strains except CDC-0381 C. auris ( $\left.\mathrm{IC}_{90}: 0.016 \mu \mathrm{g} / \mathrm{ml}\right)$ and CDC-0390 C. auris $\left(\mathrm{IC}_{90}: 0.14 \mu \mathrm{g} / \mathrm{ml}\right)$ were found to be uniformly resistant to Caspofungin showing MIC $>32 \mu \mathrm{g} / \mathrm{ml}$. Most strains except two strains of $C$. auris CDC-0383 (IC ${ }_{90}$ : > $32 \mu \mathrm{g} / \mathrm{ml})$ and CDC-0384 ( $\left.\mathrm{IC}_{90^{-}}>32 \mu \mathrm{g} / \mathrm{ml}\right)$ were found to be susceptible to another echinocandin antifungal antibiotic, Micafungin ( $\mathrm{MIC}_{50}-0.014-0.016 \mu \mathrm{g} / \mathrm{ml}, \mathrm{MIC}_{90}{ }^{-}$ $0.55 \mu \mathrm{g} / \mathrm{ml}$ ). Similar to Micafungin, all Candida strains (C. auris and C. glabrata) except strains CDC-0383 and CDC-0384 ( $\left.\mathrm{IC}_{90}->32 \mu \mathrm{g} / \mathrm{ml}\right)$ showed high susceptibility to Anidulafungin ( $\mathrm{MIC}_{50}-0.014-0.016, \mathrm{MIC}_{90}-0.24 \mu \mathrm{g} / \mathrm{ml}$ ). C. glabrata was found to be resistant to Posaconazole (no inhibition at the highest concentration $12.5 \mu \mathrm{g} /$ $\mathrm{ml}$ ). MIC of Posaconazole was based on $>50 \%$ growth inhibition although the inhibition of none of the strains reached beyond 79\% (growth inhibition range 53-79\%). The inhibitory concentration of Posaconazole for various strains ranged between 0.09-0.78 $\mathrm{\mu g} / \mathrm{ml}$. $\left(\mathrm{MIC}_{50}-0.09\right.$ $\left.0.19 \mu \mathrm{g} / \mathrm{ml} ; \mathrm{MIC}_{90}-0.78 \mu \mathrm{g} / \mathrm{ml}\right)$.

Since echinocandins are fungicidal for Candida species, the concentration at which no observed growth of $\mathrm{C}$. auris noted was taken as the fungicidal concentration. For Caspofungin, the fungicidal concentration could not be determined as all strains except one strain were resistant (>32 $\mu \mathrm{g} / \mathrm{ml})$. However, the fungicidal concentration of Micafungin (0.0156$0.125 \mu \mathrm{g} / \mathrm{ml}$ ) and Anidulafungin (0.0156-0.75 $\mu \mathrm{g} / \mathrm{ml}$ ) was evaluated based on $100 \%$ growth inhibition and culture 


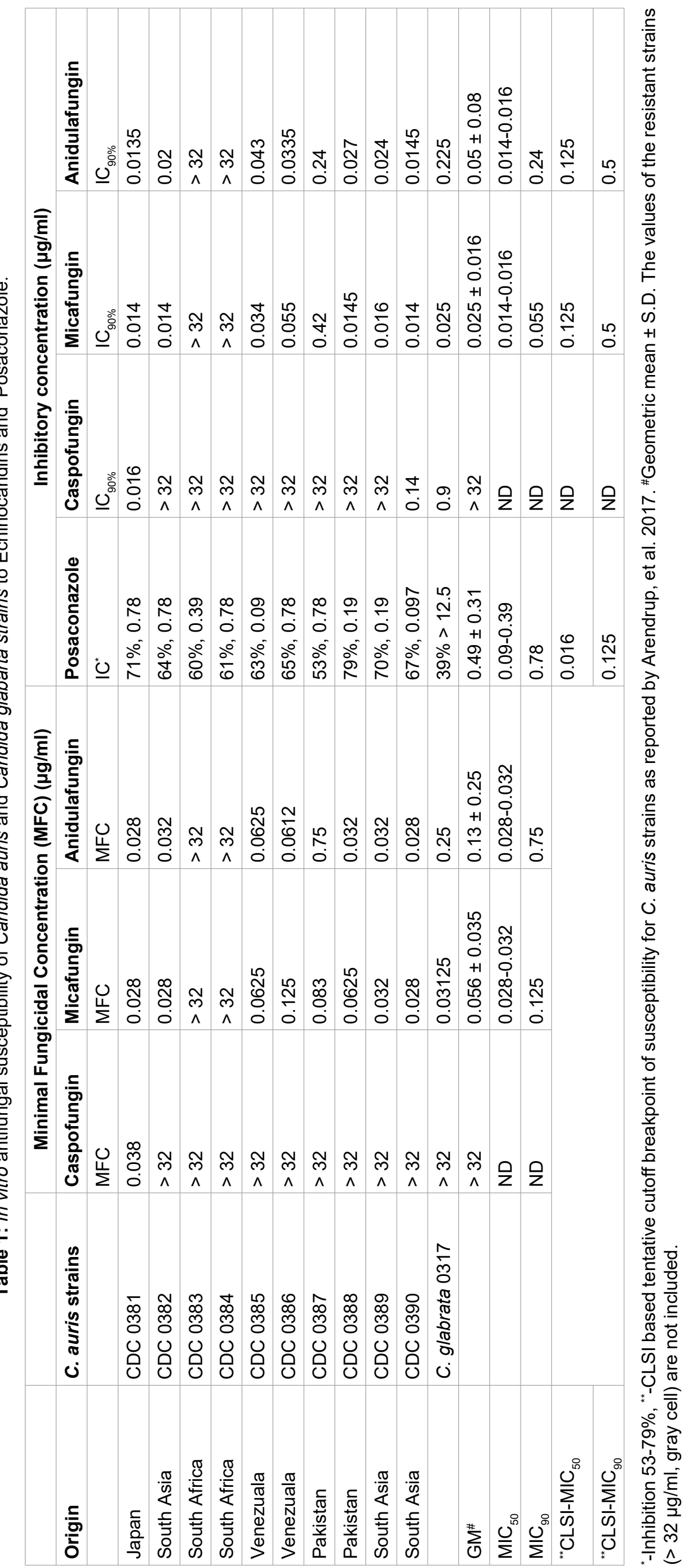


Table 2: In vitro antifungal susceptibility (fungicidal $\sim \mathrm{MIC} 100$ ) of Candida auris and Candida glabrata to Amphotericin-B (AmB) deoxycholate, and liposomal preparations of Amphotericin-B.

\begin{tabular}{|c|c|c|c|c|}
\hline \multirow[t]{2}{*}{ Origin } & \multirow[t]{2}{*}{ Candida strains } & \multirow{2}{*}{\begin{tabular}{|l|} 
AmB-deoxycholate \\
Amfocare \\
${ }^{\mathrm{M}}$
\end{tabular}} & \multicolumn{2}{|c|}{ Liposomal AmB } \\
\hline & & & AmBisome ${ }^{\circledR}$ & FUNGISOME $^{\circledR}$ \\
\hline Japan & C. auris CDC 0381 & 0.25 & 2 & 0.125 \\
\hline South Asia & C. auris CDC 0382 & 0.21 & 2 & 0.25 \\
\hline South Africa & C. auris CDC 0383 & 0.5 & 4 & 0.5 \\
\hline South Africa & C. auris CDC 0384 & 0.5 & 8 & 0.5 \\
\hline Venezuala & C. auris CDC 0385 & 1 & 16 & 0.5 \\
\hline Venezuala & C. auris CDC 0386 & 32 & $>32$ & 0.83 \\
\hline Pakistan & C. auris CDC 0387 & 32 & 32 & 0.25 \\
\hline Pakistan & C. auris CDC 0388 & 0.33 & $>32$ & 1 \\
\hline South Asia & C. auris CDC 0389 & 1 & $>32$ & 1.33 \\
\hline South Asia & C. auris CDC 0390 & 0.5 & $>32$ & 0.83 \\
\hline $\mathrm{N} / \mathrm{A}$ & C. glabrata CDC 0317 & 0.25 & 1 & 0.125 \\
\hline $\mathrm{GM}^{\#}$ & & $0.53 \pm 0.31(n=8)$ & $6.4 \pm 5.9(n=5)$ & $0.6 \pm 0.4(n=10)$ \\
\hline MIC50 & & $0.25-0.5$ & & $0.125-0.25$ \\
\hline MIC90 & & 32 & 32 & 1.3 \\
\hline${ }^{* *}$ CLSI-50 & & 0.5 & \multirow[t]{2}{*}{$\mathrm{N} / \mathrm{A}$} & \\
\hline${ }^{*}$ CLSI-90 & & 2 & & \\
\hline
\end{tabular}

N/A: Not available: * CLSI method-based tentative cutoff breakpoint of susceptibility for $C$. auris as reported by Arendrup, et al. 2017. "Geometric mean \pm S.D., the Values of resistant strains (gray cell $\geq 32$ ) are not included.

negativity. In this evaluation, two highly resistant strains (CDC0383 and CDC 0384) were not included.

Inhibitory concentrations (I.C.) for all Amphotericin-B preparations were measured at $100 \%$ growth inhibition (Table 2). Amphotericin-B deoxycholate preparation $\left(\right.$ Amfocare ${ }^{\mathrm{TM}}$ ) showed varied efficacy for all strains except CDC-0386 and CDC-0387 C. auris strains (20\%, IC $>32 \mu \mathrm{g} / \mathrm{ml}$ ) in the range of $0.25-32 \mu \mathrm{g} / \mathrm{ml}\left(\mathrm{MIC}_{50}-0.25\right.$ $0.5 \mu \mathrm{g} / \mathrm{ml}, \mathrm{MIC}_{90}-32.0 \mu \mathrm{g} / \mathrm{ml}$ ). Compared to Amfocare ${ }^{\mathrm{TM}}$, AmBisome ${ }^{\mathrm{TM}}$ showed 8-16 fold lower efficacy to inhibit five of $10 \mathrm{C}$. auris strains $\left(\mathrm{MIC}_{50} 2.0-16.0 \mu \mathrm{g} / \mathrm{ml}, \mathrm{MIC}_{90} 32\right.$ $\mu \mathrm{g} / \mathrm{ml}$ ) and one CDC-0317 C. glabrata strain (IC $1.0 \mu \mathrm{g} /$ $\mathrm{ml}$ ). Five remaining strains $(50 \%)$ of $C$. auris (CDC386CDC390) showed no inhibition of growth at the highest concentration of AmBisome ${ }^{\circ}(32 \mu \mathrm{g} / \mathrm{ml})$. Compared to these two Amphotericin-B preparations, FUNGISOME ${ }^{\circ}$ showed uniform higher efficacy by 8-32 folds. All $C$. auris strains, including those strains (CDC0383 and CDC0384), which showed uniform resistance patterns to all antifungal agents included in the present study, were completely inhibited at much lower concentrations ( MIC $_{50} 0.125-0.25 \mu \mathrm{g} / \mathrm{ml}$. MIC 90

Together, these results showed that if the conventional AFST also includes available liposomal Amphotericin-B formulations along with the traditional Amphotericin-B colloidal form, the interpretation as "resistant" susceptibility pattern of the same strain to Amphotericin-B would likely differ to "susceptible". The corrected AFST, in turn, would provide appropriate therapeutic recommendation.

\section{Discussion}

This is the first study showing a comparative susceptibility pattern of multidrug-resistant $C$. auris against two different liposomal formulations of Amphotericin-B (AmBisome ${ }^{\circ}$ and FUNGISOME ${ }^{\circ}$ ), Amphotericin-B-deoxycholate (Amfocare ${ }^{\mathrm{TM}}$ ), echinocandins, and an azole antifungal agents. Several new azoles group of antifungals, such as Voriconzole, Posaconazole, Itraconzole, Fluconazole and Isavuconazole are now available [4,30,61]. Most fungal species, including Candida, Aspergillus, Fusarium, and Mucor are resistant to Fluconazole [29,62]. Presuming so, fluconazole was not included for investigation in the present study. Since the echinocandins, such as Caspofungin, Anidualfungin, and Micafungin have shown efficacy or success rate of $50-75 \%[63,64]$ and Posaconzole $92 \%[43,65,66,79,80]$, they were included them to compare their AFST with conventional Amphotericin-B deoxycholate and two available openlabel liposomal formulations of Amphotericin-B.

Compared to the fungistatic antifungal drugs, azoles, and echinocandins, the Amphotericin-B is fungicidal and accorded distinction as a "Gold Standard" [67]. However, its high tendency to cause dose-dependent nephrotoxicity and infusion-related complications dampen its use $[38,39,68]$. Amphotericin-B-sensitive and -resistant $C$. auris strains have been recently reported in Colombia, and India and were found to be regionally restricted $[26,59]$. A recent multicenter study of AFST patterns of $C$. auris strains has shown that $\sim 8 \%$ (27 of 350 strains) displayed resistance to Amphotericin-B 
[54]. A comparison of EUCAST (European Committee for Antimicrobial Susceptibility Testing) and CLSI referencebased microdilution MICs of antifungals for several $C$. auris strains has revealed tentative cutoff values for Amphotericin-B susceptibility (CLSI-MIC $_{50}-0.5 \mu \mathrm{g} / \mathrm{ml}$, $\mathrm{MIC}_{90}-2.0 \mu \mathrm{g} / \mathrm{ml}$, EUCAST- MIC $\mathrm{C}_{50} / \mathrm{MIC}_{90}-1.0 \mu \mathrm{g} / \mathrm{ml}$ ). In all these studies, AFST patterns for Amphotericin-B were measured using the conventional Amphotericin-Bdeoxycholate. The mechanism of resistance of Candida species, including $C$. auris, to azoles has been attributed to point mutations occurring in the ergosterol biosynthesis genes (ERG11, ERG2) and to echinocandins in FKS1 gene $[54,69,70]$. Similarly, along with mutations in ergosterol biosynthesis genes [71,72], a strong association of four nonsynonymous (missense/nonsense) mutations in the protein-coding region responsible for transcription factor and membrane transport have been attributed to Amphotericin-B resistance [59]. However, this observed association does not represent the functional Amphotericin-B resistance. Several reported Amphotericin-B AFST assays are carried out with a generic form of Amphotericin-B employing the E-test or Vitek AST-YSO7 methods, however, these methods do not provide a uniform measurement of AFS pattern for Amphotericin- $B$ [26]. Hence, the present comparative study is based on the CLSI BDM method.

Resistance to Amphotericin- $B$ is rare, and thus, the development of resistance to Amphotericin-B, unlike other antifungals, has not been a significant factor in the treatment of patients [36]. To minimize inherent nephrotoxicity of Amphotericin-B in conventional colloidal formulation, liposomal Amphotericin-B (AmBisome ${ }^{\circ}$ ) and further a novel liposomal Amphotericin-B have been developed [41,44,46-48] (Table 3).

Liposomal Amphotericin-B $\quad\left(\right.$ AmBisome $\left.^{\circledR}\right)$ is composed of hydrogenated soy phosphatidylcholine (HSPC), distearoylphosphatidylglycerol (DSPG), and cholesterol [41,44]. The lipid composition, suspension medium, shape size, stability pharmacokinetics, and toxicity of this preparation substantially differ from the newer liposomal formulation, FUNGISOME ${ }^{\circledR}$ [4548]. FUNGISOME ${ }^{\circledR}$ has been proven to be safe and with high efficacy against systemic fungal infection, including candidiasis, mucrormycosis, cryptococcosis, aspergillosis, and visceral leishmaniasis [48,73-77].

Table 3: Properties of Amphotericin-B formulations.

\begin{tabular}{|c|c|c|c|}
\hline \multirow[t]{2}{*}{ Properties } & \multirow{2}{*}{$\begin{array}{l}\text { Amphotericin-B } \\
\text { deoxycholate } \\
\begin{array}{l}\text { Amfocare } \\
\mathrm{mg} / 10 \mathrm{ml}\end{array} \\
\end{array}$} & \multicolumn{2}{|l|}{ Liposomal Amphotericin-B } \\
\hline & & $\begin{array}{l}\text { AmBisome }{ }^{\circledR} \text { (Gilead) } 50 \\
\mathrm{mg} / 10 \mathrm{ml}\end{array}$ & $\begin{array}{l}\text { FUNGISOME (Lifecare Innovations } \\
1 \mathrm{mg} / \mathrm{ml} 10 \mathrm{ml}, 50 \mathrm{ml}\end{array}$ \\
\hline Particle & Micelle & $\begin{array}{l}\text { Liposome-small unilamellar } \\
\text { vesicles (SUV) }\end{array}$ & $\begin{array}{l}\text { Liposome: multilamellar vesicles MLV- } \\
\text {-- > SUV }\end{array}$ \\
\hline Size (nm) & 25 & $77.8(60-80)$ & 2743-3454-- > 20-200 \\
\hline Amphoterician B (Mol\%) & 34 & 10 & 1 \\
\hline Carrier & Deoxycholate & $\begin{array}{l}\text { Liposome ("DSPG: }{ }^{* *} \mathrm{HSPC:} \\
\text { Cholesterol) }\end{array}$ & Liposome (HSOC:Cholesterol) \\
\hline Carrier: Amphotericin B & 2:01 & (0.8:2:1):0.4(7:1::Lipid:drug) & (7:3):0.22(45:1::Lipid:drug) \\
\hline $\begin{array}{l}\text { Diateroryl phosphatidyl } \\
\text { glycerol (DSPG) }\end{array}$ & & 84 & \\
\hline $\begin{array}{l}\text { Hydrogenated Soy } \\
\text { Phosphatidyl choline } \\
\text { (HSPC) }\end{array}$ & & 213 & 31.5 \\
\hline Cholesterol & & 52 & 13.5 \\
\hline Sucrose & & 900 & \\
\hline a-tocopherol & & 0.64 & \\
\hline $\begin{array}{l}\text { Disodium succinate } \\
\text { hexahydrate }\end{array}$ & & 27 & \\
\hline Vehicle & PBS & Distilled water & Normal saline $(0.9 \% \mathrm{Nacl})$ \\
\hline Sonication & Not required & Not required & $45 \min$ \\
\hline Nephrotoxicity & $34-60 \%$ & $10-20 \%$ & Negligible or not detected \\
\hline Antifungal Clinical trials & Several-See ref. [41] & Several-See ref. $[41,42,78]$ & Limited see ref- $[41,45,48.73,75]$ \\
\hline Relative Cost/Stability & Not expensive/one week & Expensive/72h & $\begin{array}{l}\text { Less expensive } / 1 \text { year } 4^{\circ} \mathrm{C} \text {. If unused } \\
\text { Resonication after } 24 \text { hour }\end{array}$ \\
\hline Availability/usage & Universal & Universal & $\begin{array}{l}\text { Limited to certain countries. Available } \\
\text { where } C \text {. auris is most prevalent }\end{array}$ \\
\hline References & {$[41,44,47]$} & {$[40-42,46,78]$} & {$[41,45,48,73,75]$} \\
\hline
\end{tabular}


AmBisome still has higher toxicity, and hence in many previous studies, voriconazole and Posaconazole were preferred over AmBisome for the treatment of fungal infections [42,78-80]. In the unique formulation of FUNGISOME ${ }^{\oplus}$, Amphotericin-B is encapsulated in 20 $200 \mathrm{~nm}$ size predominantly unilamellar liposomes composed of phosphatidylcholine and cholesterol and stabilized in normal saline suspension. FUNGISOME ${ }^{\circ}$ is sonicated before the infusion to maximize the number of smaller liposomes [48]. Previous studies have shown that the MIC of FUNGISOME ${ }^{\star}$ is $2-16$ fold lower than the reference drug Amphotericin-B-deoxycholate against various yeasts, including Candida and Cryptococcus neoformans, dimorphic fungi, filamentous fungi, Zygomycetes, dematiaceous fungi, and dermatophytes [60]. The latter study included MIC studies of Candida spp viz. albicans, tropicalis, guillermondii, haemulonii, krusei, parapsilosis, dubliniensis, and glabrata showed susceptibility to FUNGISOME ${ }^{\circledR}$ [60]. This study, however, did not include AmBisome ${ }^{\circ}$ in the comparative AFST analysis. In another multicenter study, the MIC of AmBisome against yeasts and filamentous fungi has been reported to be 4-5 times higher than reference drug Amphotericin-B-deoxycholate [81]. However, these two susceptibility studies lack comparative analysis using $C$. auris. In this regard, the present study makes a significant contribution to $C$. auris AFST analysis and its potential application for targeted therapy.

Unlike echinocandins and azoles, all Amphotericin-B formulations - (conventional, lipid, and liposomal) have a distinctly different mechanism of drug targeting and action, pharmacokinetics, nephrotoxicity, potency, and dose as reflected in MIC. This study clearly shows that different formulations of Amphotericin-B cannot be treated the same as a generic Amphotericin- $B$ formulation. Thus, two liposomal Amphotericin-B

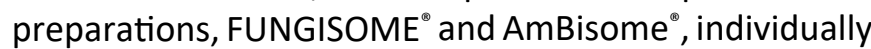
and together are different from the reference, Amphotericin-deoxycholate. As shown in Table 1 and Table 2, all MDR $C$. auris strains are susceptible to FUNGISOME ${ }^{\oplus}$. In fact, the present findings show that in comparison to AmBisome ${ }^{\circ}$, FUNGISOME ${ }^{\circ}$ showed 8 to 32-fold reduced MIC (higher efficacy) against most $C$. auris strains. Additionally, in the present study, Posaconazole and Micafungin were found to be effective against most strains, except for two strains (CDC383-CDC384) of South Africa origin. These resistant strains were found to be susceptible to FUNGISOME indicating that FUNGISOME ${ }^{\circ}$ can potentially offer substantial therapeutic benefits. Such resistant $C$. auris strains and other similar resistant fungal pathogens would be reported as "resistant" and not suited for Amphotericin-B, if the therapy is based on the conventional Amphotericin-B AFST.

It is intriguing that despitehaving knownAmphotericinB-associated dose-dependent nephrotoxicity, the liposomal formulation of FUNGISOME $^{\oplus}$ and not
AmBisome ${ }^{-}$is more effective in vitro as revealed in this study. One explanation is that the multilamellar converting to unilamellar (Table 3) structure of liposomes in the FUNGISOME preparation may allow slow release of Amphotericin-B sufficient to offer optimum fungicidal concentrations for a prolonged period providing its extended fungicidal action in vitro. Clinically, this property may remain more beneficial as its serum level below toxicity would avoid dose-dependent nephrotoxicity. Clinically, both AmBisome ${ }^{\circledR}$ and FUNGISOME ${ }^{\circledR}$ have independently shown high efficacy against fungal infection as compared to conventional Amphotericin-B deoxycholate $[48,73,82]$ although there is no clinical study that has shown side by side comparative efficacy of these two liposomal Amphotericin-B for any fungal infection including Candida auris. Amphotericin-B also serves as immunomodulator enabling the host to defend against the fungal/parasitic infection $[83,84]$. KALSOME, a FUNGISOME comparable Amphotericin-B preparation, used for leishmaniasis seems also hasimmunomodulatory and potentially protective effects [85]. At present, it is unknown whether the increased efficacy of certain liposomal Amphotericin-B preparations is mediated in part by their liposomal formulation-specific beneficiary immunomodulatory activities.

In conclusion, although the number of $C$. auris strains used in the present study are limited, they, irrespective of their origins or clad type, showed a uniform susceptibility to FUNGISOME ${ }^{\oplus}$ as compared to the other two Amphotericin-B formulations, and the pattern of susceptibility was well within the sensitive breakpoint limits [32]. This study emphasizes that the perceived notion of non-usefulness of Amphotericin-B based on its potential cytotoxic nature and/or conventional AFST for the treatment of $C$. auris infection is not justifiable and should be revisited both at laboratory assay and clinical therapy levels. Merits of liposomal Amphotericin-B preparations and their potential therapeutic applications to treat fungal infection may be universally recognized based on the improved and accurate reporting of AFST stated in the present study. The proposed modified BDAFST approach is more relevant in COVID-19 pandemic times when several fatal cases of Covid-19 patients were found to be infected with incurable fungal pathogens such as $C$. auris and other Candida spp., Aspergillus and Mucor $[27,28,86]$.

\section{Author Contributions}

Conceptualization, methodology, validation of the data, writing, editing and project administration, V. P.

\section{Funding}

This research received no external funding. The author has no financial conflict.

\section{Acknowledgments}

The author acknowledges the technical assistance of 
Mr. David Ordough during the initial phase of this study.

\section{Conflicts of Interest}

The authors declare no conflict of interest.

\section{References}

1. Lockhart SR, Guarner J (2019) Emerging and reemerging fungal infections. Semin Diagn Pathol 36: 177-181.

2. Lockhart SR (2014) Current epidemiology of Candida infection. Clin Microbiol Newsl 36: 131-136.

3. Pfaller MA, Diekema DJ, Turnidge JD, Castanheira M, Jones RN (2019) Twenty Years of the SENTRY Antifungal Surveillance Program: Results for Candida Species From 1997-2016. Open Forum Infect Dis 6: S79-S94.

4. Deorukhkar SC, Saini S, Mathew S (2014) Non-albicans Candida Infection: An Emerging Threat. Interdiscip Perspect Infect Dis 2014: 615958.

5. Bassetti M, Righi E, Costa A, Fasce R, Molinari MP, et al. (2006) Epidemiological trends in nosocomial candidemia in intensive care. BMC Infect Dis 6: 21.

6. Antinori S, Milazzo L, Sollima S, Galli M, Corbellino M (2016) Candidemia and invasive candidiasis in adults: A narrative review Eur J Intern Med 34: 21-28.

7. Antinori $S$, Milazzo L, Sollima S, Galli M, Corbellino M (2017) Critically ill patients at risk of invasive candidiasis: The "dilemma" of the best antifungal treatment strategy. Eur J Intern Med 37: e20-e21.

8. Center for Disese Control and Prevention (2015) Invasive Candidiasis.

9. Rhodes J, Fisher MC (2019) Global epidemiology of emerging Candida auris. Curr Opin Microbiol 52: 84-89.

10. Chow NA, Munoz JF, Gade L, Berkow EL, Li X, et al. (2020) Tracing the evolutionary history and global expansion of candida auris using population genomic analyses. mBio 11: e03364-e03419.

11. Kordalewska M, Perlin DS (2019) Identification of Drug Resistant Candida auris. Front Microbiol 10: 1918.

12. Cortegiani A, Misseri G, Giarratano A, Bassetti M, Eyre D (2019) The global challenge of Candida auris in the intensive care unit. Crit Care 23: 150

13. Cortegiani A, Misseri G, Chowdhary A (2019) What's new on emerging resistant Candida species. Intensive Care Med 45: 512-515.

14. Meis JF, Chowdhary A (2018) Candida auris: A global fungal public health threat. Lancet Infect Dis 18: 1298-1299.

15. Lockhart SR, Etienne KA, Vallabhaneni S, Farooqi J, Chowdhary A, et al. (2017) Simultaneous Emergence of Multidrug-Resistant Candida auris on 3 Continents Confirmed by Whole-Genome Sequencing and Epidemiological Analyses. Clin Infect Dis 64: 134-140.

16. Lockhart SR (2019) Candida auris and multidrug resistance: Defining the new normal. Fungal Genet Biol 131: 103243.

17. Chakrabarti A, Sood P (2021) On the emergence, spread and resistance of Candida auris: Host, pathogen and environmental tipping points. J Med Microbiol 70: 001318.

18. Lee WG, Shin JH, Uh Y, Kang MG, Kim SH, et al. (2011) First three reported cases of nosocomial fungemia caused by Candida auris. J Clin Microbiol 49: 3139-3142.

19. Kim MN, Shin JH, Sung H, Lee K, Kim EC, et al. (2009)
Candida haemulonii and closely related species at 5 university hospitals in Korea: Identification, antifungal susceptibility, and clinical features. Clin Infect Dis 48: e57-e61.

20. Satoh K, Makimura K, Hasumi Y, Nishiyama Y, Uchida K, et al. (2009) Candida auris sp. nov., a novel ascomycetous yeast isolated from the external ear canal of an inpatient in a Japanese hospital. Microbiol Immunol 53: 41-44.

21. Chowdhary A, Sharma C, Meis JF (2017) Candida auris: A rapidly emerging cause of hospital-acquired multidrugresistant fungal infections globally. PloS Pathog 13: e1006290.

22. Du H, Bing J, Hu T, Ennis CL, Nobile CJ, et al. (2020) Candida auris: Epidemiology, biology, antifungal resistance, and virulence. PloS Pathog 16: e1008921.

23. Rhodes J, Abdolrasouli A, Farrer RA, Cuomo CA, Aanensen DM, et al. (2018) Genomic epidemiology of the UK outbreak of the emerging human fungal pathogen Candida auris. Emerg Microbes Infect 7: 43.

24. Casadevall A, Kontoyiannis DP, Robert V (2019) On the emergence of Candida auris: Climate change, azoles, swamps, and birds. mBio 10: e01397-19.

25. Chowdhary A, Sharma A (2020) The lurking scourge of multidrug resistant Candida auris in times of COVID-19 pandemic. J Glob Antimicrob Resist 22: 175-176.

26. Kathuria S, Singh PK, Sharma C, Prakash A, Masih A, et al. (2015) Multidrug-Resistant Candida auris Misidentified as Candida haemulonii: Characterization by MatrixAssisted Laser Desorption Ionization-Time of Flight Mass Spectrometry and DNA Sequencing and Its Antifungal Susceptibility Profile Variability by Vitek 2, CLSI Broth Microdilution, and Etest Method. J Clin Microbiol 53: 18231830.

27. Hoenigl M (2020) Invasive Fungal Disease complicating COVID-19: When it rains it pours. Clin Infect Dis 73: e1645-e1648.

28. CDC (2021) Fungal diseases and Covid-19.

29. Pfaller MA, Boyken L, Hollis RJ, Kroeger J, Messer SA, et al. (2008) In vitro susceptibility of invasive isolates of Candida spp. to anidulafungin, caspofungin, and micafungin: Six years of global surveillance. J Clin Microbiol 46: 150-156.

30. Diekema DJ, Messer SA, Boyken LB, Hollis RJ, Kroeger $J$, et al. (2009) In vitro activity of seven systemically active antifungal agents against a large global collection of rare Candida species as determined by CLSI broth microdilution methods. J Clin Microbiol 47: 3170-3177.

31. Meis JF, Chowdhary A (2019) Candida auris-"Ten Years After". J Fungi (Basel) 6: 2.

32. Arendrup MC, Prakash A, Meletiadis J, Sharma C, Chowdhary A (2017) Comparison of EUCAST and CLSI Reference Microdilution MICs of Eight Antifungal Compounds for Candida auris and Associated Tentative Epidemiological Cutoff Values. Antimicrob Agents Chemother 61: e00485-17.

33. Pfaller MA, Diekema DJ, Ostrosky-Zeichner L, Rex JH, Alexander BD, et al. (2008) Correlation of MIC with outcome for Candida species tested against caspofungin, anidulafungin, and micafungin: Analysis and proposal for interpretive MIC breakpoints. J Clin Microbiol 46: 26202629.

34. Chow NA, Gade L, Tsay SV, Forsberg K, Greenko JA, et al. (2018) Multiple introductions and subsequent transmission 
of multidrug-resistant Candida auris in the USA: A molecular epidemiological survey. Lancet Infect Dis 18: 1377-1384.

35. Rex JH, Walsh TJ, Sobel JD, Filler SG, Pappas PG, et al (2000) Practice guidelines for the treatment of candidiasis. Infectious Diseases Society of America. Clin Infect Dis 30: $662-678$

36. Ellis D (2002) Amphotercin B: Spectrum and resistance. J Antimicrob Chemother 49: S1-7-S1-10.

37. Kshirsagar NA, Gokhale PC, Pandya SK (1995) Liposomes as drug delivery system in leishmaniasis. J Assoc Physicians India 43: 46-48.

38. Wingard JR, Kubilis P, Lee L, Yee G, White M, et al. (1999) Clinical significance of nephrotoxicity in patients treated with amphotericin B for suspected or proven aspergillosis. Clin Infect Dis 29: 1402-1407.

39. Bates DW, Su L, Yu DT, Chertow GM, Seger DL, et al (2001) Mortality and costs of acute renal failure associated with amphotericin B therapy. Clin Infect Dis 32: 686-693.

40. Tiphine M, Letscher Bru V, Herbrecht R (1999) Amphotericin $B$ and its new formulations: Pharmacologic characteristics, clinical efficacy, and tolerability. Transpl Infect Dis 1: 273283.

41. Dupont B (2002) Overview of the lipid formulations of amphotercin B. J Antimicrob Chemother 49: 31-36.

42. Walsh TJ, Pappas P, Winston DJ, Lazarus HM, Petersen F, et al. (2002) Voriconazole compared with liposomal amphotericin B for empirical antifungal therapy in patients with neutropenia and persistent fever. N Engl J Med 346: 225-234.

43. Torres HA, Hachem RY, Chemaly RF, Kontoyiannis DP, Raad II (2005) Posaconazole: A broad-spectrum triazole antifungal. Lancet Infect Dis 5: 775-785.

44. Patel R (2000) Amphotericin B colloidal dispersion. Exp Opin Pharmacother 1: 475-488.

45. Kshirsagar NA, Pandya SK, Kirodian GB, Sanath S (2005) Liposomal drug delivery system from laboratory to clinic. J Postgrad Med 51: S5-S15.

46. Adler-Moore JP, Gangneux JP, Pappas PG (2016) Comparison between liposomal formulations of amphotericin B. Med Mycol 54: 223-231.

47. Faustino C, Pinheiro L (2020) Lipid Systems for the Delivery of Amphotericin B in Antifungal Therapy. Pharmaceutics 12: 29.

48. Sanath SS, Gogtay NJ, Kshirsagar NA (2005) Postmarketing study to assess the safety, tolerability and effectiveness of Fungisome: An Indian liposomal amphotericin B preparation. J Postgrad Med 51: S58-S63.

49. Clancy CJ, Nguyen MH (1999) Correlation between in vitro susceptibility determined by $E$ test and response to therapy with amphotericin B: Results from a multicenter prospective study of candidemia. Antimicrob. Agents Chemother 43: 1289-1290.

50. Nguyen MH, Clancy CJ, Yu VL, Yu YC, Morris AJ, et al. (1998) Do in vitro susceptibility data predict the microbiologic response to amphotericin $B$ ? Results of a prospective study of patients with Candida fungemia. J Infect Dis 177: 425430.

51. Rex JH, Cooper CR, Jr, Merz WG, Galgiani JN, Anaissie EJ (1995) Detection of amphotericin B-resistant Candida isolates in a broth-based system. Antimicrob. Agents Chemother 39: 906-909.
52. Wanger A, Mills K, Nelson PW, Rex JH (1995) Comparison of Etest and National Committee for Clinical Laboratory Standards broth macrodilution method for antifungal susceptibility testing: Enhanced ability to detect amphotericin B-resistant Candida isolates. Antimicrob Agents Chemother 39: 2520-2522.

53. Rex JH, Pfaller MA, Walsh TJ, Chaturvedi V, Espinel-Ingroff A, et al. (2001) Antifungal susceptibility testing: Practical aspects and current challenges. Clin Microbiol Rev 14: 643658.

54. Chowdhary A, Prakash A, Sharma C, Kordalewska M, Kumar A, et al. (2018) A multicentre study of antifungal susceptibility patterns among 350 Candida auris isolates (2009-17) in India: Role of the ERG11 and FKS1 genes in azole and echinocandin resistance. J Antimicrob Chemother 73: 891-899.

55. Chowdhary A, Anil KV, Sharma C, Prakash A, Agarwal K, et al. (2014) Multidrug-resistant endemic clonal strain of Candida auris in India. Eur J Clin Microbiol Infect Dis 33: 919-926.

56. Bassetti M, Taramasso L, Nicco E, Molinari MP, Mussap M, et al. (2011) Epidemiology, species distribution, antifungal susceptibility and outcome of nosocomial candidemia in a tertiary care hospital in Italy. PLoS One 6: e24198.

57. Clinical and laboratory Standard Institute (2008) Reference Method for Broth Dilution Antifungal Susceptibility Testing of Yeasts, in 3rd/4th informal Supplement. CLSI M27-A3/ A4: Clinical and Laboratory Standards Institute, Wayne, PA.

58. Law D, Moore CB, Denning DW (1997) Amphotericin $B$ resistance testing of Candida spp: A comaprison of methods. J Antimicrob Chemother 40: 109-112.

59. Escandon P, Chow NA, Caceres DH, Gade L, Berkow EL, et al. (2019) Molecular Epidemiology of Candida auris in Colombia Reveals a Highly Related, Countrywide Colonization with Regional Patterns in Amphotericin B Resistance. Clin Infect Dis 68: 15-21.

60. Rudramurthy SM, Jatana M, Singh R, Chakrabarti A (2013) In vitro antifungal activity of Indian liposomal amphotericin $B$ against clinical isolates of emerging species of yeast and moulds, and its comparison with amphotericin B deoxycholate, voriconazole, itraconazole and fluconazole. Mycoses 56: 39-46.

61. Diekema DJ, Messer SA, Hollis RJ, Boyken LB, Tendolkar S, et al. (2007) Evaluation of Etest and disk diffusion methods compared with broth microdilution antifungal susceptibility testing of clinical isolates of Candida spp. against posaconazole. J Clin Microbiol 45: 1974-1977.

62. Pfaller MA, Boyken L, Hollis RJ, Kroeger J, Messer SA, et al. (2009) In vitro susceptibility of clinical isolates of Aspergillus spp. to anidulafungin, caspofungin, and micafungin: A head-to-head comparison using the CLSI M38-A2 broth microdilution method. J Clin Microbiol 47: 3323-3325.

63. Sinnollareddy MG, Roberts JA, Lipman J, Akova M, Bassetti M, et al. (2015) Pharmacokinetic variability and exposures of fluconazole, anidulafungin, and caspofungin in intensive care unit patients: Data from multinational Defining Antibiotic Levels in Intensive care unit (DALI) patients Study. Crit Care 19: 33.

64. Bassetti M, Merelli M, Righi E, Diaz-Martin A, Rosello EM, et al. (2013) Epidemiology, species distribution, antifungal susceptibility, and outcome of candidemia across five sites in Italy and Spain. J Clin Microbiol 51: 4167-4172. 
65. Greer ND (2003) Voriconazole: The newest triazole antifungal agent. Proc (Bayl Univ Med Cent) 16: 241-248.

66. Greer ND (2007) Posaconazole (Noxafil): A new triazole antifungal agent. Proc (Bayl Univ Med Cent) 20: 188-196.

67. Ostrosky-Zeichner L, Marr KA, Rex JH, Cohen SH (2003) Amphotericin B: Time for a new "gold standard". Clin Infect Dis 37: 415-425.

68. Bates DW, Su L, Yu DT, Chertow GM, Seger DL, et al. (2001) Correlates of acute renal failure in patients receiving parenteral amphotericin B. Kidney Int 60: 1452-1459.

69. Hull CM, Bader O, Parker JE, Weig M, Gross U, et al. (2012) Two clinical isolates of Candida glabrata exhibiting reduced sensitivity to amphotericin $B$ both harbor mutations in ERG2. Antimicrob Agents Chemother 56: 6417-6421.

70. Hull CM, Parker JE, Bader O, Weig M, Gross U, et al. (2012) Facultative sterol uptake in an ergosterol-deficient clinical isolate of Candida glabrata harboring a missense mutation in ERG11 and exhibiting cross-resistance to azoles and amphotericin B. Antimicrob Agents Chemother 56: 4223-4232.

71. Vandeputte P, Tronchin G, Larcher G, Ernoult E, Berges T, et al. (2008) A nonsense mutation in the ERG6 gene leads to reduced susceptibility to polyenes in a clinical isolate of Candida glabrata. Antimicrob Agents Chemother 52: 37013709.

72. Vandeputte $P$, Tronchin G, Berges T, Hennequin C, Chabasse D, et al. (2007) Reduced susceptibility to polyenes associated with a missense mutation in the ERG6 gene in a clinical isolate of Candida glabrata with pseudohyphal growth. Antimicrob Agents Chemother 51: 982-990.

73. Bodhe PV, Kotwani RN, Kirodian BG, Kshirsagar NA Pandya SK (2002) Open label, randomised, comparative phase III safety and efficacy study with conventional amphotericin B and liposomal amphotericin B in patients with systemic fungal infection. J Assoc Physicians India 50: 662-670.

74. Gokhale PC, Kshirsagar NA, Khan MU, Pandya SK, Meisheri YV, et al. (1994) Successful treatment of resistant visceral leishmaniasis with liposomal amphotericin B. Trans R Soc Trop Med Hyg 88: 228.

75. Kotwani RN, Bodhe PV, Kirodian BG, Mehta KP, Ali US, et al. (2003) Treatment of neonatal candidiasis with liposomal amphotericin B (L-AMP-LRC-1): Phase II study. Indian Pediatr 40: 545-550.

76. Kotwani RN, Gokhale PC, Bodhe PV, Kirodian BG, Kshirsagar NA (2001) Safety and efficacy of liposomal amphotericin $B$ in patients with cryptococcal meningitis. $J$ Assoc Physicians India 49: 1086-1090.

77. Bodhe PV, Kotwani RN, Kirodian BG, Pathare AV, Pandey AK, et al. (1999) Dose-ranging studies on liposomal amphotericin B (L-AMP-LRC-1) in the treatment of visceral leishmaniasis. Trans R Soc Trop Med Hyg 93: 314-318.

78. Herbrecht R, Denning DW, Patterson TF, Bennett JE, Greene RE, et al. (2002) Voriconazole versus amphotericin $\mathrm{B}$ for primary therapy of invasive aspergillosis. $\mathrm{N}$ Engl $\mathrm{J}$ Med 347: 408-415.

79. Herbrecht R (2004) Posaconazole: A potent, extendedspectrum triazole anti-fungal for the treatment of serious fungal infections. Int J Clin Pract 58: 612-624.

80. Herbrecht R (2004) Voriconazole: Therapeutic review of a new azole antifungal. Expert Rev Anti Infect Ther 2: 485497.

81. Jessup C, Reyes G, Fothergill A, McCarthy D, Rinaldi M, et al. (2000) A head-on comparison of the in vitro antifungal activity of conventional and lipid-based amphotericin B: A multicenter study. J Chemother 12: 22-29.

82. Adler-Moore J, Lewis RE, Bruggemann RJM, Rijnders BJA, Groll AH, et al. (2019) Preclinical Safety, Tolerability, Pharmacokinetics, Pharmacodynamics, and Antifungal Activity of Liposomal Amphotericin B. Clin Infect Dis 68: S244-S259.

83. Mesa-Arango AC, Scorzoni L, Zaragoza O (2012) It only takes one to do many jobs: Amphotericin $B$ as antifungal and immunomodulatory drug. Front Microbiol 3: 286.

84. Sangalli Leite F, Scorzoni L, Mesa Arango AC, Casas C, Herrero E, et al. (2011) Amphotericin B mediates killing in Cryptococcus neoformans through the induction of a strong oxidative burst. Microbes Infect 13: 457-467.

85. Shadab Md, Jha B, Asad M, Deepthi M, Kamran M, et al. (2017) Apoptosis-like cell death in Leishmania donovani treated with KalsomeTM10, a new liposomal amphotericin B. PloS One 12: e0171306.

86. Rudramurthy SM, Hoenigl M, Meis JF, Cornely OA, Muthu $V$, et al. (2021) ECMM/ISHAM recommendations for clinical management of COVID-19 associated mucormycosis in low- and middle-income countries. Mycoses 64: 1028-1037. 